\title{
Cellular automata simulation of traffic flow through a road with a construction zone
}

\author{
E. Kita, T. Tamaki \& H. Shimizu \\ Graduate School of Information Sciences, \\ Nagoya University, Nagoya, Japan
}

\begin{abstract}
In this paper, we discuss the cellular automata simulation of the traffic flow on a two-lane road with a construction zone. The simulation model is based on the cellular automata and the stochastic velocity model. In the stochastic velocity model, the vehicles are controlled with a uniform random number. A two-lane road with a construction zone is considered as the object domains and the simulation results are compared with the results of the one-lane and two-lane roads without a construction zone. Although it has been predicted from the analytical study that the traffic flow on a road with a construction zone depends only on the number of the available lanes, the simulation results shows that the traffic flow depends not only on the number of the available lanes but also the length of the construction zone.
\end{abstract}

\section{Introduction}

The existing traffic simulation models can be briefly classified into the macroand micro-models $[1,2,3]$. In the macro-model, the traffic flow is considered as the liquid flow and, therefore represented with the constitutive equation. In the micro-model, the movement of each vehicle is simulated with a computer and the emergent phenomenon of driving vehicles shows the traffic flow. Since the computational cost of the macro-model is much lower than that of the micromodel, the macro-model simulation is often adopted for the large-scale traffic simulation in which a large number of vehicles are driving in a wide area. Tanahashi et al. have pointed out that it is very important to represent the different cartelistic feature of each vehicle for more accurate simulation of the traffic flow [3]. So, the authors have focused on the cellular automata model for the 


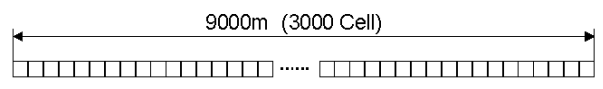

Figure 1: One-lane road.

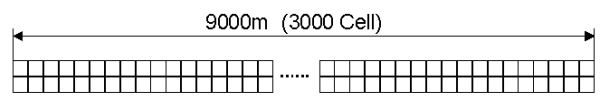

Figure 2: Two-lane road.

traffic flow simulation because the cellular automata model can represent well the different cartelistic features of the vehicles.

A first cellular automata model for the traffic flow simulation is Rule-184 CA model [4]. After that, Nagel-Schrekenberg (NaSch) model and the vehicle following models have been presented $[5,6]$. However, in the above models, the velocity is represented with the number of cells which the vehicle passes through at each time step. Since a vehicle driving very fast passes through a lot of cells for each time step, the vehicle may pass over the other stopping vehicle or the crosssection when a traffic signal is red. For overcoming the difficulty, the authors have presented the stochastic velocity model. Since, in the model, the movement of the vehicle is up to one cell at each time step, the rules for the vehicle movement can be simplified. In this paper, the cellular automata simulation based on the stochastic velocity model will be applied to the traffic flow on a two-lane road with a construction zone.

\section{Simulation model}

\subsection{Object domain under consideration}

Consider as the object domain under consideration, a one-lane, a two-lane or a three-lane highway roads. Each road is represented with 1000 square cells of $3 \mathrm{~m}$ wide and $3 m$ length. The cell representation of a one-lane, a two-lane and a threelane roads are shown in Figs. 1 and 2, respectively. Each vehicle is represented with two adjoin cells. In the simulation, one time-step means $0.1 s$ in real time.

\subsection{Stochastic velocity model}

The stochastic velocity model represents the movement of the vehicle driving with a velocity $\bar{v}\left(<v_{\max }\right)$ and the maximum velocity $v_{\max }$ as follows.

1. A threshold $P_{0}$ is estimated by

$$
P_{0}=\frac{\bar{v}}{v_{\max }} .
$$

2. An uniform random number $P(x)$ is generated from 0 to 1 .

WIT Transactions on The Built Environment, Vol 89,(C) 2006 WIT Press

www.witpress.com, ISSN 1743-3509 (on-line) 


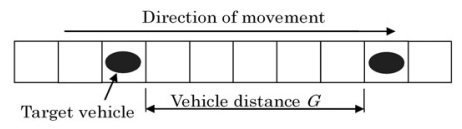

Figure 3: Vehicle distance in a one-lane road.

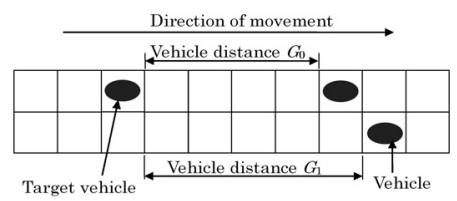

Figure 4: Vehicle distance in two-lane road.

3. If $P(x)<P_{0}$, the vehicle moves by one cell.

\subsection{Basic local rules}

The basic local rule is classified into the movement and the velocity local rules. In the movement local rule, the vehicle moves according to the concept of the stochastic velocity model. The velocity local rule changes the velocity according to the distance between the vehicle and its forehand one.

\subsubsection{Estimation of vehicle distance}

In a one-lane road, the estimation of the vehicle distance is explained in Fig. 3. The number of cells between the target vehicle and its forehand vehicle is counted and the distance $G$ is calculated as the product of the cell size $(3 \mathrm{~m})$ and the number of cells.

In a two-lane road, the estimation of the vehicle distances is explained in Fig. 4. The distance $G_{0}$ is the distance from the forehand vehicle on the lane which the target vehicle drives. The distance $G_{1}$ is the distance from the forehand vehicle on the other lane than that which the target vehicle drives.

\subsubsection{Safety vehicle distance}

In the study, it is assumed that the vehicles are going to keep the safety distance from the forehand vehicle. The vehicle distance and its safety vehicle distance are referred as $G$ and $G_{s}$, respectively. The Japanese automobile inspection manual tells that the vehicle driving at a velocity $v$ should keep the distance from the forehand vehicle as

$$
G_{s}^{0}=0.15 \times v+0.0097 \times v^{2} .
$$

The vehicle distance depends not only on the velocity but also the characteristic features of vehicles and the drivers. So, the minimum value of the vehicle distance $G_{s}^{\text {min }}$ is specified randomly for each vehicle. When the vehicle stops, $G_{s}=0$. 


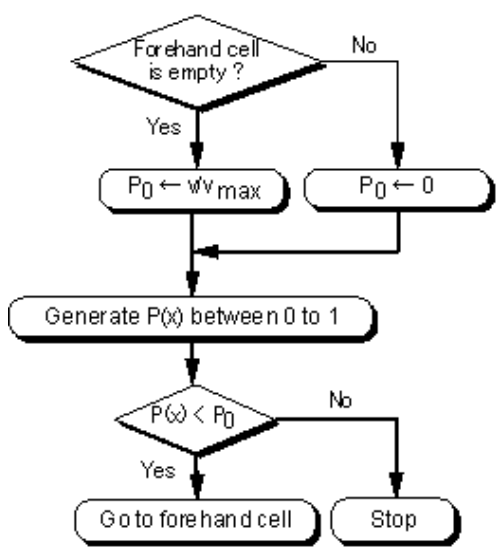

Figure 5: Movement local rule for through vehicle.

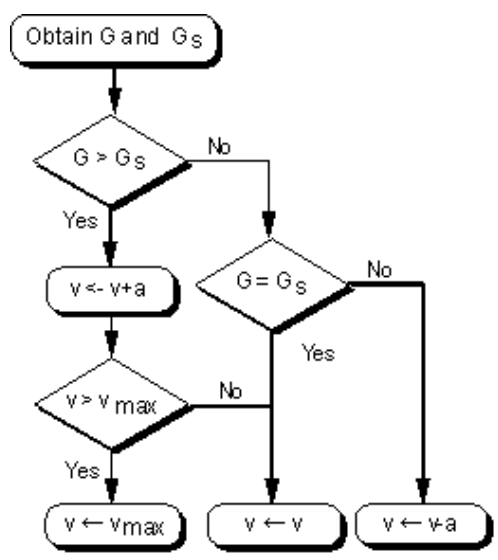

Figure 6: Velocity local rule.

The safety vehicle distance $G_{s}$ is defined as

$$
G_{s}=\left\{\begin{array}{cc}
\max \left(G_{s}^{0}, G_{s}^{\min }\right) & (v>0) \\
0 & (v=0)
\end{array}\right.
$$

where $\max \left(G_{s}^{0}, G_{s}^{\min }\right)$ means the bigger one between $G_{s}^{0}$ and $G_{s}^{\min }$.

\subsubsection{Movement local rule for through vehicle}

The movement local rule is shown in Fig. 5. We shall consider that a vehicle on Cell A moves to Cell B. If Cell B is empty, the vehicle moves according to the stochastic velocity model described in section 2.2. If Cell B is occupied with the other one, the vehicle waits on Cell A at the time step. 


\subsubsection{Velocity local rule}

The velocity local rule is shown in Fig. 6 . The parameters $v_{\max }$ and $\alpha$ indicates the maximum velocity and the acceleration of the vehicle. In the numerical example, the acceleration $\alpha$ is specified according to the Land, Infrastructure and Transportation Ministry of Japan [7].

The velocity $v$ is changed from the relationship between the safety vehicle distance $G_{s}$ and the actual vehicle distance $G$. If $G_{s}=G$, the velocity is not changed. If $G_{s}>G, v \leftarrow v-\alpha$ for decreasing the velocity to broaden the distance. If $G_{s}<G, v \leftarrow v+\alpha$ for increasing the velocity to shorten the distance. Finally, if $v>v_{\max }, v \leftarrow v_{\max }$.

\subsection{Behavior local rule}

A vehicle behavior is also controlled according to the distance from the forehand vehicle. The estimation of the vehicle distance is explained first and then, the behavior local rules on a one-lane, two-lane and three-lane roads are defined.

\section{(1) Behavior local rule on a one-lane road}

1. Estimate the vehicle distance $G$.

2. Calculate the safety distance $G_{s}$ by equation (3) from a vehicle velocity $v$.

3. Go to the velocity and then, the movement local rules.

\section{(2) Behavior local rule on a two-lane road}

1. Estimate the vehicle distance $G_{0}$ and $G_{1}$.

2. Calculate the safety distance $G_{s}$ by equation (3) from the vehicle velocity $v$.

3. If $G_{0}>G_{1}$, then $G \leftarrow G_{0}$.

4. If $G_{0}<G_{1}$, then $G \leftarrow G_{1}$.

5. Go to the velocity and then, the movement local rules.

\subsection{Definition of construction cells}

The construction road is represented with the "construction cells". The vehicles can not pass over the construction cells.

\section{Numerical examples}

\subsection{In case of short construction zone}

A two-lane road with a short construction zone is considered as a numerical example (Fig. 7). Each lane is composed of 3000 cells $(9000 \mathrm{~m})$ and the construction zone is of two cells $(6 \mathrm{~m})$.

Vehicles go from the left end to right end and the periodic boundary conditions are specified on both ends. In the periodic boundary condition, the vehicles running off from the right end are coming in from the left end. The use of the periodic boundary condition can keep the vehicle density to the constant value. The 


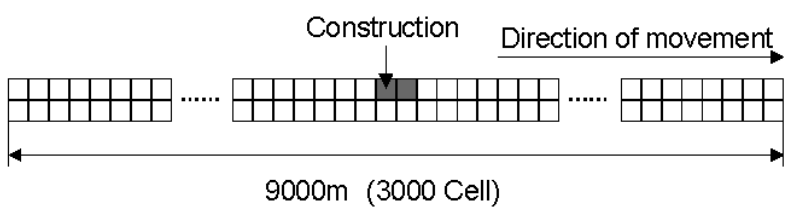

Figure 7: Two-lane road including short construction zone.

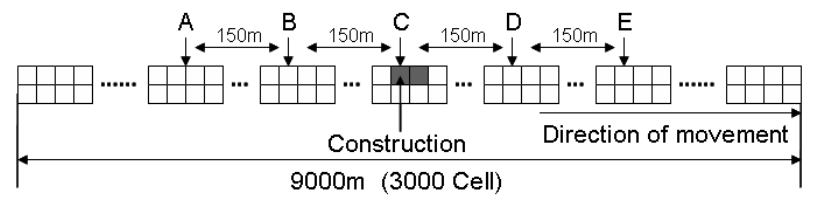

Figure 8: Placement of observation points.

parameters are specified as one time step $=0.1 \mathrm{~s}$, maximum velocity $=80 \mathrm{Km} / \mathrm{h}$, acceleration rate $=0.6 \mathrm{~m} / \mathrm{s}^{2}$, and minimum safety following vehicle distance $=$ $0 \mathrm{~m}$. Five observation points are located uniformly along the road to evaluate the traffic flow amount and the mean velocity (Fig. 8).

Figure 9 illustrates the traffic flow amount at the observation points as well as that on the road without a construction zone. The abscissa denotes the vehicle occupancy defined as the percentage of the cells occupied with vehicles in all cells. The ordinate indicates the traffic flow amount estimated as the number of the vehicles passing the observation point per one hour. The traffic flow amounts at five observation points show similar tendency. The traffic flow amount increases gradually according to the increase of the vehicle occupancy and reaches to the maximum value at the vehicle occupancy of $0.03(3 \%)$. The traffic flow amount keeps its maximum value at the vehicle occupancy of 0.03 to 0.7 . When the occupancy is greater than 0.7 , the traffic flow amount is identical to that at the road including a construction zone. Besides, the maximum traffic flow amount at the two-lane road with a construction zone is half as many as that at the two-lane road without a construction zone. A theoretical study of the traffic flow amount on the road with a construction zone has pointed out that the maximum traffic flow amount depends on that at the construction zone. The present results indicates the same conclusion.

\subsubsection{Effect of length of construction zone}

A two-lane road with a construction zone is considered as a numerical example. Each lane is composed of 3000 cells (Fig. 10). The length of the construction zone is 300 cell $(900 \mathrm{~m})$, which is much longer than that in the section 3.1. The parameters are the same as that in the section 3.1. Five observation points are located along the road. 


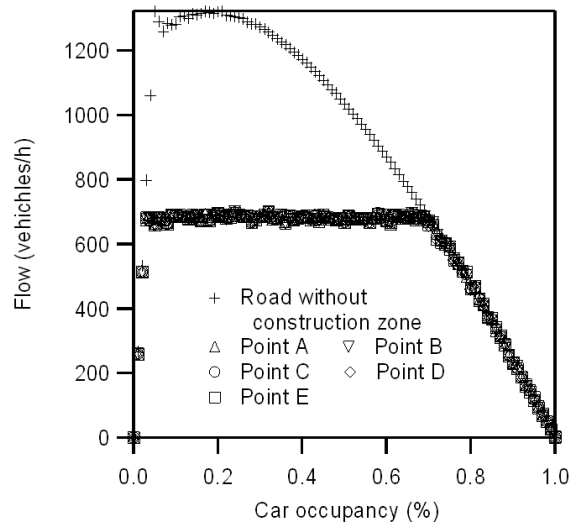

Figure 9: Traffic flow and vehicle occupancy (Short construction zone).

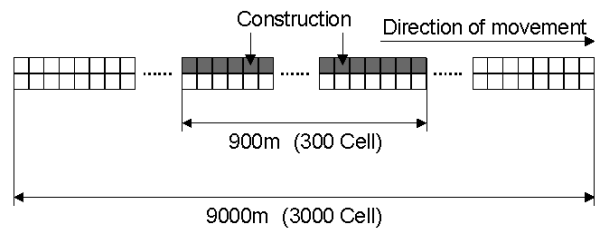

Figure 10: A Two-lane road woth a long construction zone.

Figure 11 shows the traffic flow amount versus the vehicle occupancy. The traffic flow amount is the average value of them at five observation points. The traffic flow amount at the short congestion zone is also shown in the same figure. The labels indicate as follows.

Case 1 A one-lane road without a construction zone.

Case 2 A two-lane road without a construction zone.

Case 3 A two-lane road with a short $(6 \mathrm{~m})$ construction zone.

Case 4 A two-lane road with a short $(900 \mathrm{~m})$ construction zone.

In the case of a long construction zone, the traffic flow amount keeps the maximum value at the occupancy of 0.03 to 0.7 , which is very similar to the case of a short construction zone. As pointed out by the results, the maximum traffic flow amount in a long construction zone is fewer than that in a short construction zone and moreover, the maximum traffic flow amount in a long construction zone is equal to that in a one-lane road without a construction zone. This results indicate that the traffic flow amount on the road with a construction zone depends on not only the number of the available lanes at construction zone but also the length of the construction zone.

Figure 12 shows the traffic flow amount versus the length of the construction zone. The object domain is shown in Fig. 10 and the vehicle occupancy is taken 


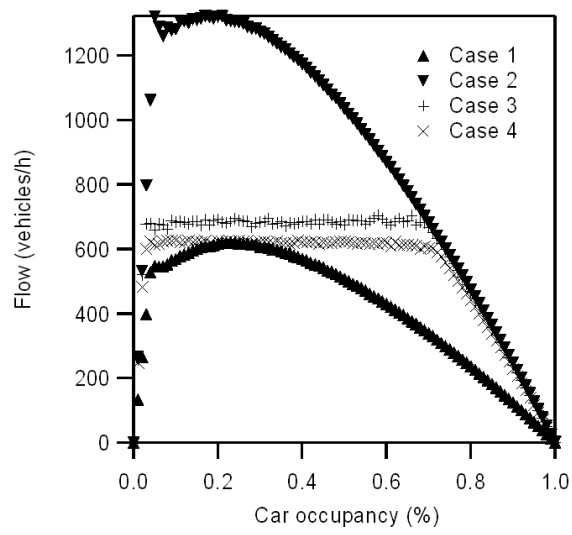

Figure 11: Traffic flow and vehicle occupancy (Long construction zone).

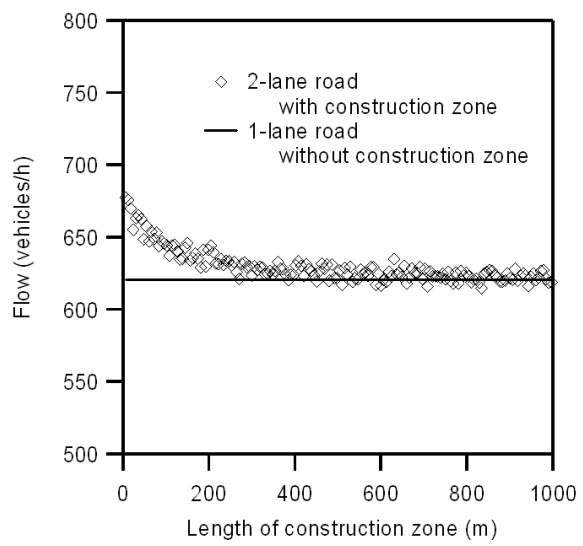

Figure 12: Effect of construction zone length to traffic flow.

as the value at which the maximum traffic flow amount can be observed in the one-lane road. The abscissa and the ordinate denotes the length of the construction zone and the traffic flow amount, respectively. The traffic flow amount on the onelane road is shown by a line. Figure 12 indicates that the traffic flow amount on the two-lane road converges the maximum traffic flow amount on the one-lane road according to the increase of the length of the construction zone.

\section{Conclusions}

This paper describes the application of the traffic flow simulation using the cellular automata and the stochastic velocity model to the traffic flow through the road with a construction zone. 
The traffic flow on a two-lane road with a short construction zone has been simulated. The results illustrated that the maximum traffic flow amount depended on the maximum traffic flow amount on the construction zone. A traffic congestion occurred at the construction zone and developed to a downstream of the traffic flow.

Next, the effect of the length of the construction zone has been discussed on a two-lane road. Comparing with the traffic flow amount on a one-lane road indicated that the traffic flow amount on a two-lane road with a short construction zone was greater than that on a one-lane road without a construction zone and that the traffic flow amount on a two-lane road with a construction zone converged to that on a one-lane road according to the increase of the length of the construction zone.

\section{References}

[1] i Transport Lab. City traffic flow simulation model AVENUE. http://www.itransportlab.jp/products/avenue/.

[2] Toyota Central Laboratoy. NETSTREAM. http://www.tytlabs.co.jp/ office/library/lib_01/netstream/.

[3] I. Tanahashi, H. Kitaoka, M. Baba, H. Mori, S. Terada, and E. Teramoto. Wide area traffic flow simulator NETSTREAM. In IPSJ, ITS, Vol. 9-2, pp. 9-14, 2002. (in Japanese).

[4] S. Wolfram. Cellular Automata and Complexity. Adison-Wesley Publishing Company, 1 edition, 1994.

[5] K. Nagel and M. Schreckenberg. Cellular automaton model for freeway traffic. Journal of Physics I france, Vol. 2, pp. 2221-2229, 1992.

[6] Y. Sugiyama. Optimal velocity model for traffic flow. Computer Physics Communications, Vol. 121-122, pp. 399-401, 1999.

[7] Infrastructure Land and Transportation Ministry of Japan. List of fuel consumption rate of vehicles (in Japanese). 